\title{
Highlights from the 58th Annual Scientific Sessions of the American College of Cardiology, March 28 to 31, 2009, Orlando, Florida
}

\author{
Frederic S. Resnic, MD, MSc, FACC, and Akshay Desai, MD, MPH, FACC
}

The 58th Annual Scientific Sessions of the American College of Cardiology (ACC), held March 28 through 31, 2009, in Orlando, Florida, provided a forum for the initial release of clinical trial data as well as comprehensive review of many topics of significant interest to cardiothoracic surgeons.

\section{CORONARY ARTERY DISEASE AND CORONARY STENTS}

Quality of Life With Stents Versus Surgery in Treatment of Multivessel and Left Main Coronary Artery Disease

The SYNTAX investigators explored the quality of life and cost-effectiveness of the strategy of percutaneous coronary intervention (PCI) versus coronary artery bypass grafting $(\mathrm{CABG})$ for left main or multivessel coronary artery disease in this international randomized study of over 1600 patients. The group had previously published the 1-year outcomes from the study, which demonstrated similar rates of death and myocardial infarction at 12 months, with increased rates of repeat revascularization for the patients treated with PCI. ${ }^{1}$ Dr David Cohen reported that although angina relief was slightly better with CABG than drug-eluting stents (DES) at 6 and 12 months, the differences were very small, and the overall costs (including hospitalization and rehabilitation) were significantly higher at 1 year for the patients treated with CABG. The 1-year cost-effectiveness (based on US health care costs) significantly favored PCI for patients with low and moderate levels of angiographic complexity; CABG was economically attractive for patients with the most severe angiographic complexity (SYNTAX score $>$ 32). Another cost-effectiveness analysis of the SYNTAX patients is planned when the 5-year follow-up data become available, which should allow some insight into the longterm cost-effectiveness of CABG versus PCI for patients with both multivessel coronary artery disease as well as left main coronary artery disease. ${ }^{2}$

\section{IMPROVING SAFETY AND OUTCOMES OF CORONARY STENTS}

Several studies presented during ACC 2009 confirmed the safety of DES as compared with traditional bare metal stents

\footnotetext{
From the Brigham and Women's Hospital and Harvard Medical School, Boston, Mass. Address for reprints: Frederic S. Resnic, MD, MSc, FACC, Brigham and Women's Hospital, Division of Cardiology, 75 Francis Street, Tower 3B, Boston, MA 02115 (E-mail: fresnic@partners.org).

J Thorac Cardiovasc Surg 2009;138:795-7

$0022-5223 / \$ 36.00$

Copyright (c) 2009 by The American Association for Thoracic Surgery doi:10.1016/j.jtcvs.2009.06.008
}

(BMS). The largest of these analyses was based on the ACC National Cardiovascular Data Registry (NCDR) dataset through linkage of case records to the Medicare claims database to analyze clinical outcomes up to 30 months after stent implantation. This study compared the clinical outcomes of over 260,000 patients treated at more than 650 centers throughout the United States between January 2004 and December 2006 and demonstrated a $25 \%$ reduction in adjusted rates of death or myocardial infarction in the patients treated with DES as compared with those treated with BMS. The benefit of DES was sustained to 30 months following stent implantation, reinforcing the relative safety of DES as compared with BMS. ${ }^{3}$

Two trials were reported that evaluated the impact of acute (as a loading dose) statin therapy, administered immediately prior to PCI, on the rates of periprocedural myocardial infarction. The NAPLES II investigators randomized 668 patients who were scheduled to have elective PCI and who were not on statin therapy already to receive an 80 $\mathrm{mg}$ atorvastatin loading dose 24 hours prior to the procedure versus placebo. The study found that patients receiving the atorvastatin loading dose had a $44 \%$ reduction in the rates of postprocedural myocardial infarction (defined as creatinine kinase MB $>3 \times$ upper limit of normal). ${ }^{4}$ The ARMYDA-RECAPTURE investigators explored the benefits of reloading high-dose statins immediately prior to PCI in patients on chronic statin therapy. The study found a $63 \%$ reduction in the combined major cardiovascular events and a $44 \%$ reduction in the risk of periprocedural myocardial infarction in the 352 patients randomized to receive a reloading dose of a statin therapy in the trial. ${ }^{5}$

\section{HEART FAILURE AND SURGICAL VENTRICULAR REMODELING}

The Hypothesis 2 Substudy of the Surgical Treatment for Ischemic Heart Failure (STICH) trial was designed to test the proposition that surgical venticular remodeling (SVR) added to $\mathrm{CABG}$ is superior to $\mathrm{CABG}$ alone for the management of patients with coronary artery disease (left ventricular ejection fraction $\leq 35 \%$ ) and a dominant region of anterior akinesis or dyskinesis suitable for treatment with SVR. The primary outcome of the trial was a composite of death from any cause or hospitalization for cardiac causes. One thousand patients were recruited from 96 medical centers in 23 countries. Despite a statistically greater reduction in left ventricular size with SVR relative to CABG alone, over a median 48 months of follow up, no difference was observed in the occurrence of the primary outcome between the CABG group and the 
combined procedure group. The 30-day surgical rates of death for $\mathrm{CABG}$ alone $(5 \%)$ and for the combined procedure $(6 \%)$ were similar and low overall. Although key patient subgroups were examined for a treatment interaction, no subset experiencing benefit from SVR + CABG over CABG alone was identifiable. Overall, these results emphasize that the routine addition of SVR to CABG in patients with reduced ejection fraction and coronary artery disease is not beneficial. Though the specific reasons for discrepancy between the results of this randomized trial experience and the prior observational data are unclear, the data do highlight that the remodeling benefits of neurohormonal antagonism cannot be replicated or augmented through direct reduction of left ventricular volumes in the operating room. The value of CABG (with or without SVR) relative to medical therapy alone in patients with coronary artery disease and reduced ejection fraction is the focus of the ongoing Hypothesis 1 Subgroup of the STICH trial. ${ }^{6}$

\section{CATHETER-BASED TREATMENT FOR VALVULAR HEART DISEASE}

The treatment of calcific aortic stenosis using catheterbased aortic valve prostheses remains an area of intense interest within the cardiovascular community, with several live cases broadcast demonstrating the evolving technologies throughout the ACC 2009 meeting and multiple plenary sessions dedicated to the exploration of these devices and techniques. Two technologies were the focus of demonstrations: the Edwards Sapien balloon-expandable transcatheter valve and the CoreValve self-expanding stented valve system. The international experience and outcomes in several thousand patients implanted with each device were reviewed and discussed at ACC-2009. In the United States, the pivotal randomized trial of the Edwards valve, the PARTNER study, is approaching completion of enrollment, and initial results are anticipated in 2010. The US pivotal trial for the Medtronic CoreValve system is anticipated to begin within the next 12 months in the United States, pending approval of final trial design by Food and Drug Administration (FDA).

\section{STRATEGIES TO REDUCE STROKE RISK IN ATRIAL FIBRILLATION \\ Alternative Medication Strategies to Prevent Stroke in Atrial Fibrillation}

Although vitamin $\mathrm{K}$ antagonists (such as warfarin) reduce the risk of stroke in patients with atrial fibrillation, nearly one-half of such patients are judged by their physicians to be unsuitable for this therapy and are treated with aspirin alone. The Atrial Fibrillation Clopidogrel Trial with Irbesartan for Prevention of Vascular Events (ACTIVE-A) trial randomized 7554 patients with atrial fibrillation and 1 or more additional risk factors for stroke and who were deemed unsuitable for warfarin therapy to treatment with clopidogrel
$(75 \mathrm{mg} / \mathrm{d}$ ) or placebo in addition to aspirin (recommended dose, 75-100 mg) over a median follow-up of 3.6 years. The primary outcome, a composite of major vascular events including stroke, myocardial infarction, systemic embolism, or death from vascular causes, was reduced by $11 \%$ with the combination of clopidogrel and aspirin relative to aspirin alone, a reduction powered in large part by a highly significant $28 \%$ reduction in stroke. This benefit accrued at the expense of a substantial increase in major bleeding with clopidogrel and aspirin in combination $(2.0 \%$ per year) relative to aspirin alone (1.3\% per year). ${ }^{7,8}$

These data highlight that the addition of clopidogrel provides incremental reduction in vascular events over treatment with aspirin alone at the cost of more bleeding. Although warfarin remains the treatment of choice for patients with atrial fibrillation at high risk for stroke (data from ACTIVE-W arm of the same program highlighted that therapy with a vitamin $\mathrm{K}$ antagonist reduces the rate of stroke by $42 \%$ relative to therapy with clopidogrel plus aspirin $^{9}$ ), these data provide support for a potential alternative to aspirin monotherapy in the large proportion of patients who are thought to be ineligible for warfarin therapy.

\section{LEFT ATRIAL APPENDAGE OCCLUSION DEVICE TO PREVENT STROKE IN PATIENTS WITH ATRIAL FIBRILLATION}

Among patients with atrial fibrillation who are eligible for warfarin therapy, PROTECT-AF trial examined the role of percutaneous device closure of the left atrial appendage (LAA) as a therapeutic alternative to warfarin therapy. The study randomized 800 patients with nonvalvular atrial fibrillation eligible for warfarin therapy from 59 enrolling centers in the United States and Europe in a 2:1 fashion to therapy with the Watchman device (Atritech, Plymouth, Minn), an LAA occluder placed distal to the ostium of the LAA to occlude flow and prevent the migration of thrombus, or long-term warfarin in a noninferiority design. The device is delivered via a catheter placed in the left atrium via transseptal catheterization. Although initially also treated with warfarin to allow for endothelialization, $87 \%$ of patients in the device arm were able to discontinue warfarin at 45 days. After 900 patient-years of follow-up, the strategy of LAA occlusion was associated with a statistically significant reduction in hemorrhagic stroke relative to warfarin and was noninferior to warfarin with regard to all-cause stroke and all-cause mortality. However, safety events, particularly pericardial effusion, were increased by twofold in the device group. ${ }^{10}$ (Editor's note: The Watchman device received recommendation for approval from the medical review panel of the FDA on April 23, 2009.)

\section{PREVENTIVE CARDIOLOGY DEVELOPMENTS}

The JUPITER trial randomized 17,802 healthy men and women with both low-density lipoprotein (LDL) cholesterol 
levels of less than $130 \mathrm{mg}$ per deciliter and high-sensitivity C-reactive protein (hsCRP) levels of $2.0 \mathrm{mg}$ per liter or higher to receive rosuvastatin (Crestor) or placebo. The trial was stopped early at a median follow-up of 1.9 years due to a $44 \%$ reduction in the combined rate of myocardial infarction, stroke, arterial revascularization, unstable angina, or death from cardiovascular causes in the rosuvastatin-treated patients. ${ }^{11}$ A key new analysis of the JUPITER data presented at ACC 2009 demonstrated that lowering both LDL and C-reactive protein results in higher event-free survival than either intervention alone. Specifically, Ridker and colleagues noted that patients in the trial achieving both an LDL cholesterol $<70 \mathrm{mg} / \mathrm{dL}$ and hsCRP $<2 \mathrm{mg} / \mathrm{L}$ experienced a $65 \%$ reduction in vascular events relative to a $33 \%$ reduction among those who achieved only one or neither target. For patients initiating drug therapy for prophylaxis of cardiovascular disease, reductions in both LDL cholesterol and hsCRP appear to be important indicators of the success of treatment with statin therapy. ${ }^{12}$

\section{DEVELOPMENTS IN CARDIAC SURGICAL DEVICE RESEARCH}

Dr Davy Cheng presented a meta-regression analysis of endovascular (TEVAR) versus open repair of thoracic aortic disease due to trauma, dissection, or degenerative conditions. The analysis of 42 studies, including 5888 patients treated, demonstrated a significant reduction in overall complications with TEVAR as compared with open surgical repair, with a reduction in death at 30 days of $56 \%$, which was sustained to 3 years. Other end points including renal dysfunction following intervention and transfusion requirements were also reduced in the patients treated with TEVAR as compared with the patients having open surgery. ${ }^{13}$ Therefore, these data indicate that, in the absence of prospective randomized trial data, TEVAR appears to have clinical advantages over open surgical repair in the patient groups included in the analyzed studies.

The results of the VentrAssist (VentraCore, New South Wales, Australia) implantable ventricular assist device (VAD) bridge to transplant pivotal trial was reported by $\mathrm{Dr}$ Andrew Boyle. Among the first 98 patients treated with this centrifugal VAD, $85 \%$ of patients either were alive with the pump or had been transplanted successfully, exceeding the predefined performance goal for this pilot study. In addition, treated patients experienced significant improvements in quality of life after implantation of the VentrAssist device. These results point to the VentrAssist centrifugal VAD emerging as another mechanical support option as a bridge to cardiac transplantation. ${ }^{14}$

\section{References}

1. Serruys P, Maurice MC, Kappetein P, et al. Percutaneous coronary intervention versus coronary-artery bypass grafting for severe coronary artery disease. N Engl J Med. 2008;360:961-72.

2. Holmes D. PROTECT AF trial: randomized prospective trial of percutaneous LAA closure vs. warfarin for stroke prevention in AF. American College of Cardiology 2008 Scientific Sessions; Late Breaking Clinical Trials. March 28, 2009; Orlando, Fla.

3. Douglas P. Outcomes following coronary stenting: a national study of long term, real-world outcomes of bare-metal and drug-eluting stents. American College of Cardiology 2008 Scientific Sessions; Late Breaking Clinical Trials. March 28, 2009; Orlando, Fla.

4. Brigouri C. Impact of Loading Dose of Atorvastatin on Periprocedural Myocardial Infarction (NAPLES II). American College of Cardiology 2008 Scientific Sessions; Late Breaking Clinical Trials. March 30, 2009; Orlando, Fla.

5. DiSciascio G. Efficacy of Atorvastatin Reload in Patients on Chronic Statin Therapy Undergoing Percutaneous Coronary Intervention (ARMYDA-RECAPTURE). American College of Cardiology 2008 Scientific Sessions; Late Breaking Clinical Trials. March 30, 2009; Orlando, Fla

6. Jones RH, Velazquez EJ, Michler RE, et al. Coronary bypass surgery with or without surgical ventricular reconstruction. N Engl J Med. 2009;360:1705-17.

7. Connolly SJ. Clopidogrel Plus Aspirin vs. Aspirin Alone for Prevention of Vascular Events in Patients with Atrial Fibrillation at High Risk of Stroke (ACTIVE A).American College of Cardiology 2008 Scientific Sessions; Late Breaking Clinical Trials. March 31, 2009; Orlando, Fla.

8. ACTIVE Investigators. Effect of clopidogrel added to aspirin in patients with atrial fibrillation. $N$ Engl J Med. 2009;360:2066-78.

9. ACTIVE Writing Group of the ACTIVE Investigators. Clopidogrel plus aspirin versus oral anticoagulation for atrial fibrillation in the Atrial fibrillation Clopidogrel Trial with Irbesartan for prevention of Vascular Events (ACTIVE W): a randomised controlled trial. Lancet. 2006;367:1903-12.

10. Holmes D. Percutaneous Left Atrial Appendage Closure vs. Warfarin for Stroke Prevention in Atrial Fibrillation (PROTECT AF). American College of Cardiology 2008 Scientific Sessions; Late Breaking Clinical Trials. March 28, 2009; Orlando, Fla.

11. Ridker PM, Danielson E, Fonseca FAH, et al. Rosuvastatin to prevent vascular events in men and women with elevated C-reactive protein. $N$ Engl J Med. 2008;359:2195-207.

12. Ridker PM, Danielson E, Fonseca FAH, et al. Reduction in C-reactive protein and LDL cholesterol and cardiovascular event rates after initiation of rosuvastatin: a prospective study of the JUPITER trial. Lancet. 2009;373:1175-82.

13. Cheng D. Endovascular vs. Open Surgical Repair of Thoracic Aortic Disease (TEVAR). American College of Cardiology 2008 Scientific Sessions; Late Breaking Clinical Trials. March 30, 2009; Orlando, Fla.

14. Boyle A. The VentrAssist U.S. Pivotal Bridge to Cardiac Transplantation Trial. American College of Cardiology 2008 Scientific Sessions; Late Breaking Clinical Trials. March 30, 2009; Orlando, Fla. 